\title{
The Later Evolution of the Contact Binaries AP Leo, AK Her, AB And and AM Leo
}

\author{
L. Li, Z. Han, F. Zhang \\ Yunnan Observatory, National Astronomical Observatories, CAS, \\ Kunming, Yunnan Province, P. R. China
}

\begin{abstract}
A detailed study of the periods and light curves of binaries AK Her, AP Leo, AB And and AM Leo is presented. Based on the study of the $O-C$ curves, we find that the period variation of each system contains several components with different frequencies, and we suggest that the periodical variations in the periods are likely influenced by different mechanisms. Based on the study of the light curve changes, we find that the light curves exhibit two kinds of variations: rapid variations and long-term variations. We investigate the physical mechanisms which may underlie the variations of the period and the light curve of each system and obtain some new conclusions. According to the characteristics of the rapid light variation in these systems, we suggest that the rapid change in the light curve is probably caused by pulsation of the common envelope, and that the mechanism(s) causing the pulsation may be mass transfer through the inner Lagrangian point $\mathrm{L}_{1}$ or its variation. Finally, the evolutionary trends of these systems are discussed, and we suggest that these systems may be progenitors of cataclysmic variables.
\end{abstract}

\section{Introduction}

W UMa systems have been divided by Binnendijk (1970) into A- and W-type systems according to whether the primary minimum in the light curve is a transit, in which the smaller star partially eclipses the larger, or an occultation, when the larger star is in front. W-type systems generally exhibit unstable light curves and variable orbital periods, and the light curve synthesis indicates that their common envelopes are very shallow. The systems AK Her, AP Leo, $\mathrm{AB}$ And, and AM Leo belong to W-type W UMa-type contact binaries, and their orbital periods and light curves are highly variable.

It is widely believed that W UMa binaries and cataclysmic variables (CV) are genetically related objects, in particular, that CV systems are evolutionary remnants of contact binaries, a suggestion first put forward by Sahade (1959) and Kraft (1962). This connection rests principally upon circumstantial evidence, namely, the similarity in total masses, periods, velocity dispersions, and space distributions of these two classes of objects (Kraft 1962, 1965, 1967; Artiukhina 1964; Kraft \& Luyten 1965). Moreover, no third class of binaries is known which shares these properties with contact systems and CVs. A physical picture has been proposed to describe the transition from contact to CV-type 


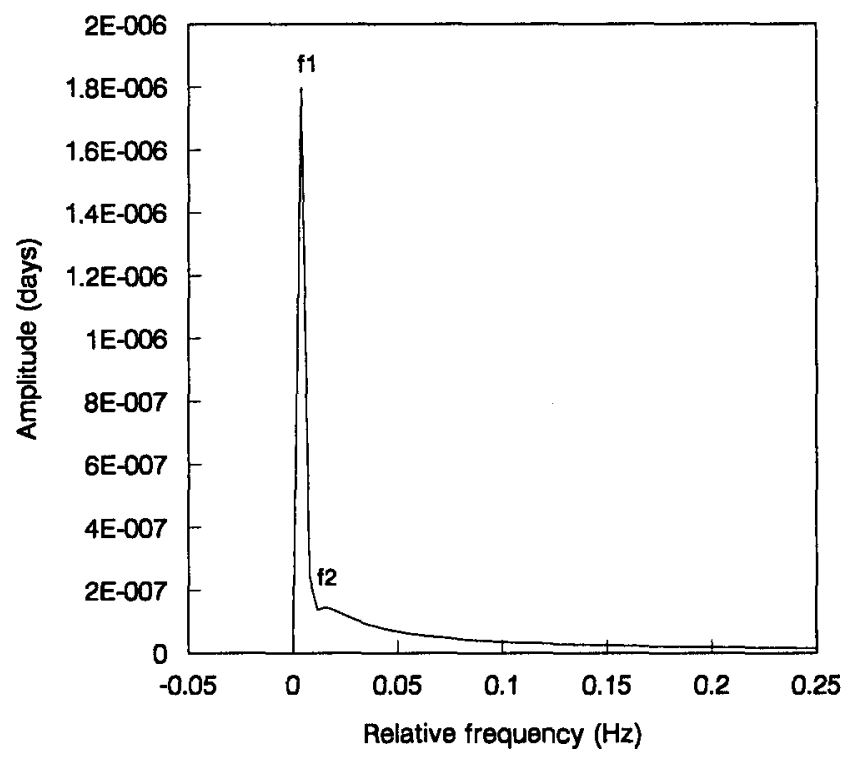

Figure 1. The spectrum of the function $P(E)$ of $\mathrm{AB}$ And.

system by Meyer \& Meyer-Hofmeister (1979). In this paper, we will discuss the evolutionary trends of these systems.

\section{Period Changes}

The orbital periods of these systems change rapidly. Detailed period studies for AK Her and AP Leo were performed recently by $\mathrm{Li}$ et al. (2001) and $\mathrm{Li}$ et al. (2002), respectively. We investigate the orbital periods of the systems $\mathrm{AB}$ And and AM Leo again using a method proposed by Kalimeris et al. (1994a, $1994 \mathrm{~b})$. We calculate the period functions $P(E)$ of $\mathrm{AB}$ And and AM Leo, and obtain their power spectra through a spectral analysis of the period functions. The power spectra of the period functions of $\mathrm{AB}$ And and AM Leo are plotted in Figs. 1 and 2, respectively. It is seen from Fig. 1 that the two periodical components correspond to periods of $85.5 \mathrm{yr}$ and $24.1 \mathrm{yr}$ for $\mathrm{AB}$ And. As seen from Fig. 2, the two periodical components correspond to periods of $36.4 \mathrm{yr}$ and $9.7 \mathrm{yr}$ for AM Leo. The component with the longer period may be caused by the light-time effect due to the influence of a third body. A mechanism based on magnetic activity cycles has been proposed by Matese \& Whitmire (1983), Applegate \& Patterson (1987), and Applegate (1989). The component with the shorter period may be caused by magnetic activity cycles. These results may be published elsewhere. 


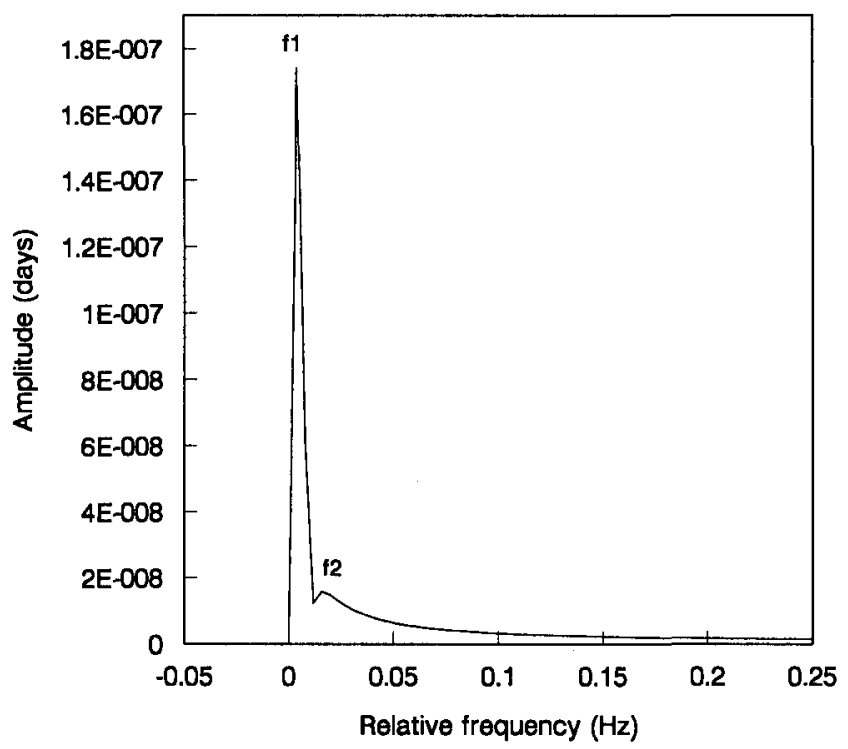

Figure 2. The spectrum of the function $P(E)$ of AM Leo.

\section{Light Curve Changes}

The light curves of these systems are very unstable. The light curve shows two kinds of variations with different time scales in each of these systems. One is a rapid light variation (i.e. night to night variation). The light curves of these systems exhibited a larger scatter, and the scatter is obviously larger than their observational errors. The scatter generally has an amplitude of $0.02-0.04 \mathrm{mag}$ with a time scale of a few hours or a few days. In these systems, the scatter is especially apparent near the two minima and the two maxima in their light curves. The amplitude and the time scale of this kind of light variation are very similar to those of pulsating variables. The mechanism causing this kind of light variation has been proposed by $\mathrm{Li}$ et al. $(2001,2002)$. It may be caused by the pulsation of the common envelope due to mass transfer or its variation. Unfortunately, most of W UMa-type binaries are very faint, and the resolution of their spectra is very low at present. We have no proof of the pulsation of the common envelope in the spectrum. It is therefore highly desirable to obtain spectra with spectral and time resolutions sufficiently high to reflect the pulsation of the common envelopes of W UMa systems using a large telescope.

The other one is a long-term change of the light curve. The detailed studies of the long-term light variations for AK Her and AP Leo have been made by $\mathrm{Li}$ et al. (2001) and $\mathrm{Li}$ et al. (2002), respectively. The long-term light variation of AB And was found by Demircan et al. (1994), and they showed this kind of light variation in their Fig. 5. We add a data point in the $V$ filter observed by Parimucha et al. (2000) in 1999. The light variation in the $V$ filter as a function of time is plotted in Fig. 3. The long-term light variation of AM Leo was found 


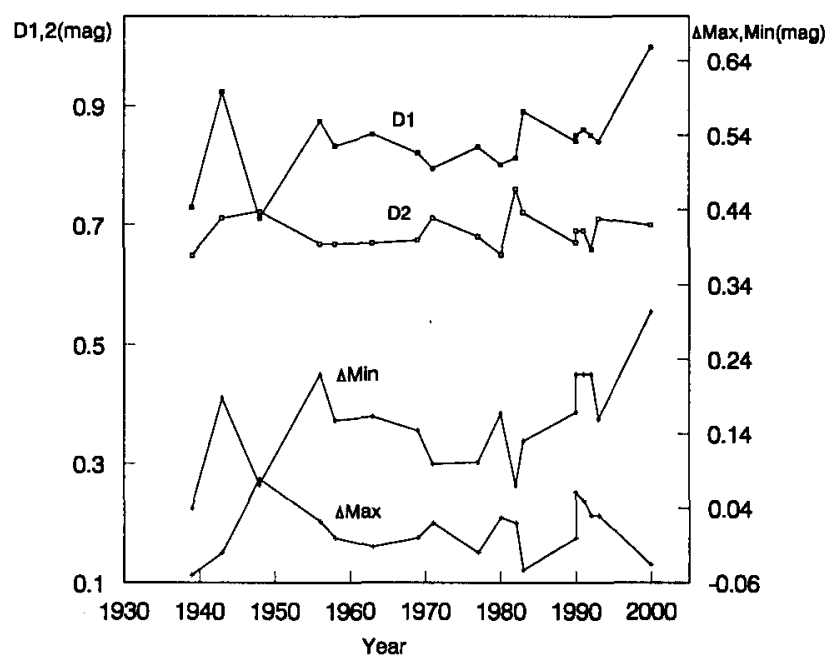

Figure 3. Variation of the depths of the primary and the secondary minima $\left[D_{1,2}=\operatorname{mag}(M i n I, I I)-\operatorname{mag}(M a x I)\right]$, and the magnitude difference between the two minima $[\Delta M i n=\operatorname{mag}(M i n I)-$ $\operatorname{mag}(\operatorname{MinII})]$, and the two Maxima $[\Delta M a x=\operatorname{mag}(\operatorname{Max} I)-$ $\operatorname{mag}(\operatorname{Max} I I)]$ for $\mathrm{AB}$ And.

by Demircan \& Derman (1992). The light variation in the $V$ filter as a function of time is plotted in Fig. 4. The characteristics of the variations in the $B$ filter are almost the same as those in $V$ in both systems. It is seen in Figs. 3 and 4 that the variations of $D 1$ (the depth of the primary eclipse) and $D 2$ (the depth of the secondary one) vary with different directions. When the depth of the primary eclipse, $D 1$, decreases, the depth of the secondary, $D 2$, increases, and vice visa. The time scale of this kind of light variation is about $20 \mathrm{yr}$ for $\mathrm{AB}$ And and $10 \mathrm{yr}$ for AM Leo. It is very similar to the shorter time scale periodical variation of the orbital period of each system, which is caused by magnetic activity cycles. Based on the analysis, we obtain that the correlation of the variations in $D 1$ and $D 2$ is caused by magnetic activity cycles.

\section{Summary and Discussion}

According to the observed properties mentioned above, we can infer the evolutionary progresses of these systems. The light curves and the orbital periods of these systems are highly variable, implying that these systems are very unstable. The periods vary rapidly. Once the variation of the orbital period is fast enough, the rotation of the common envelope cannot catch up with that of the internal binary, and can give rise to an asynchronous rotation between the common envelope and the internal binary. According to the theory of Meyer \& Meyer-Hofmeister (1979), the asynchronous rotation can produce 'frictional 


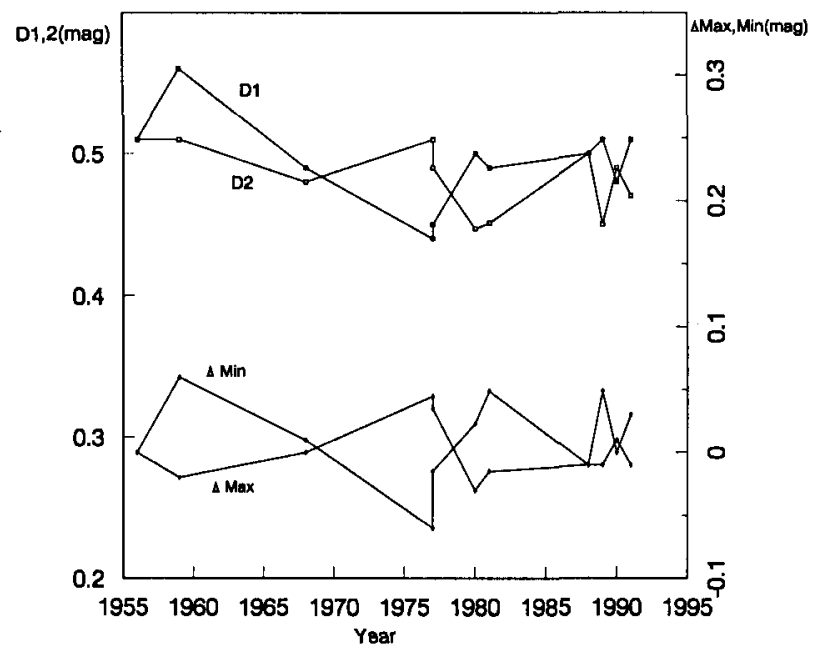

Figure 4. Variations of the depths of the primary and the secondary minima $\left[D_{1,2}=\operatorname{mag}(\operatorname{MinI}, I I)-\operatorname{mag}(\operatorname{MaxI}, I I)\right]$, and the magnitude difference between the two minima $[\Delta M i n=\operatorname{mag}(M i n I)-$ $\operatorname{mag}(\operatorname{MinII})]$, and the two Maxima $[\Delta M a x=\operatorname{mag}(\operatorname{Max} I)-$ $\operatorname{mag}(M a x I I)]$ for AM Leo

luminosity' in the differential rotating region, and lead to the transfer of the angular momentum from the internal binary to the common envelope. The frictional luminosity adds to the luminosity of the system and increases the total luminosity. Because of the transfer of the angular momentum, the period of the binary system will decrease. If the total luminosity increases, the common envelope can expand, and the internal binary may collapse, and the luminosity of the nuclear reaction will increase. If so, the total luminosity of the binary system will increase rapidly. The rapid increase of the total luminosity would probably produce an expansion wave or even an explosive wave. These waves may lead to the loss of the common envelope. These conclusions are consistent with a scenario in which these systems evolve into the cataclysmic variables in the future.

Acknowledgments. We are grateful to the support from the Chinese National Science Foundation (Grant No. 19925312) and from the 973 Scheme (NKBRSF G19990754).

\section{References}

Applegate, J. H. 1989, ApJ, 337, 865

Applegate, J. H., \& Patterson, J. 1987, ApJ, 322, L99

Artiukhina, N. M. 1964, Perem. Zvezdy, 15, 127 
Binnendijk, L. 1970, Vistas Ast., 12, 217

Demircan, O., \& Derman, E. 1992, AJ, 103, 593

Demircan, O., Derman, E., Akalin, A., Selam, S., \& Müyesseroğlu, Z. 1994, MNRAS, 267, 19

Kalimeris, A., Rovithis-Livaniou, H., \& Rovithis, P. 1994a, A\&A, 282, 775

Kalimeris, A., Rovithis-Livaniou, H., Rovithis, P., Oprescu, G., Dumitrescu, A., \& Suran, M. D. 1994b, A\&A, 291, 765

Kraft, R. P. 1962, ApJ, 135, 408

Kraft, R. P. 1965, ApJ, 142, 1588

Kraft, R. P. 1967, PASP, 79, 395

Kraft, R. P., \& Luyten, W. J. 1965, ApJ, 142, 1041

Li, L., Zhang, F., \& Han Z. 2001, A\&A, 368, 595

Li, L., Zhang, F., \& Han Z. 2002, PASJ, 54, 73

Matese, J. J., \& Whitmire, D. P. 1983, A\&A, 117, L7

Meyer, E., \& Meyer-Hofmeister, E. 1979, A\&A, 78, 167

Parimucha, S., Pribulla, T., \& Vanko, M. 2000, IBVS, 4834

Sahade, J. 1959, Mem. Soc. Roy. Sci. Liège, 5-ième Ser., 3, 76 